Supporting Information for

\title{
Zwitterionic Ru(III) Complexes: Stability of Metal-Ligand Bond and Host-Guest Binding with Cucurbit[7]uril
}

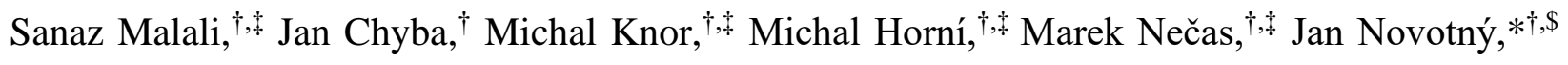
Radek Marek*t,‡\$\$

${ }^{\dagger}$ CEITEC - Central European Institute of Technology, Masaryk University, Kamenice 5, CZ62500 Brno, Czechia

$\$$ Department of Chemistry, Faculty of Science, Masaryk University, Kamenice 5, CZ-62500 Brno, Czechia

\$National Center for Biomolecular Research, Faculty of Science, Masaryk University, Kamenice 5, CZ-62500 Brno, Czechia

* Corresponding Authors

jan.novotny@ceitec.muni.cz (J.N.), radek.marek@ ceitec.muni.cz (R.M.) 


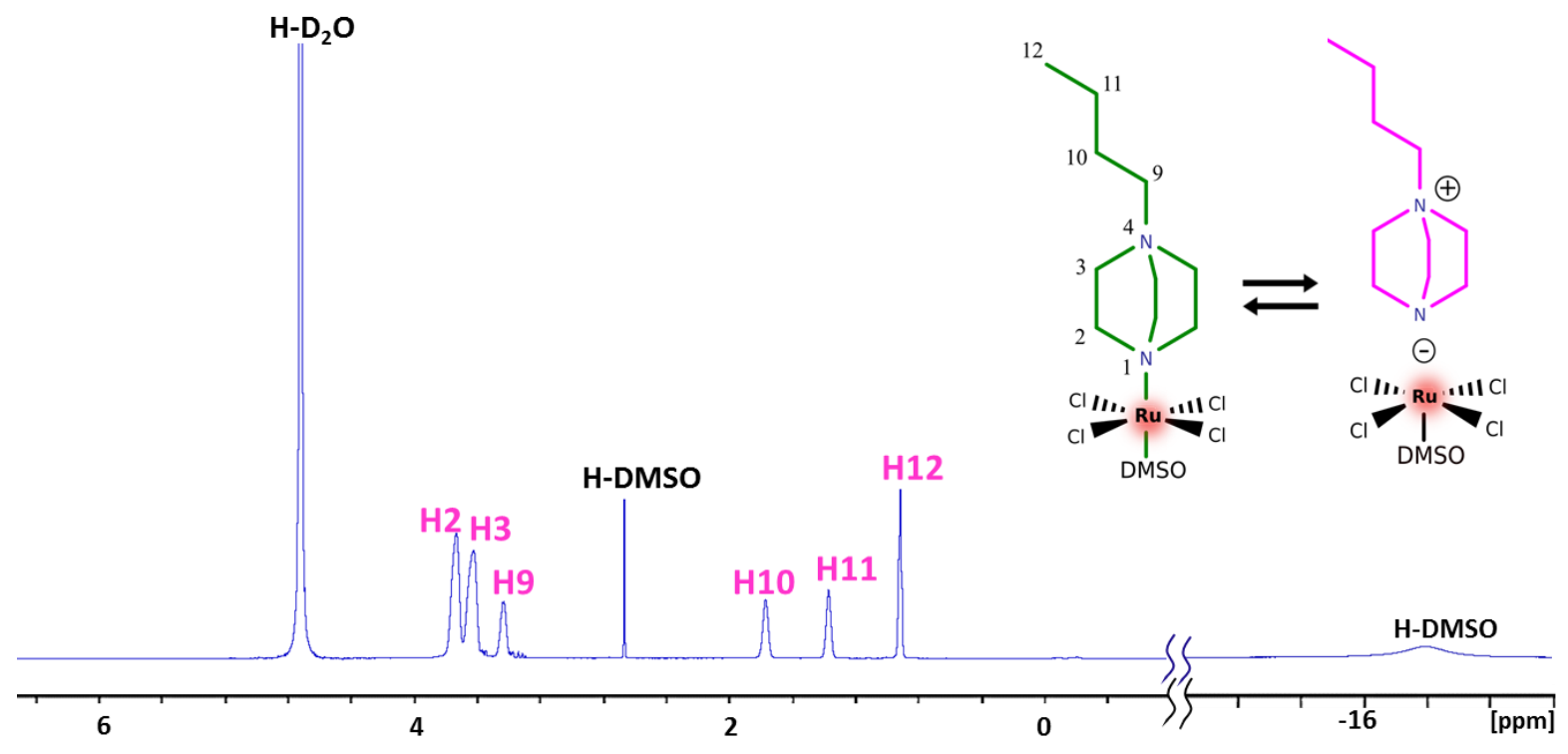

Figure S1. ${ }^{1} \mathrm{H}$ NMR spectrum of compound 1a dissolved in $\mathrm{D}_{2} \mathrm{O}$ at $298 \mathrm{~K}$. The polar water environment stabilizes the ion pair formed by the dissociation of zwitterionic compound 1a. The DABCO-R ligand in the ion pair is not exposed directly to the paramagnetic environment of the $\mathrm{RuCl}_{4} \mathrm{DMSO}$ core in contrast to that in 1a. Color code: green - zwitterionic system 1a (not detected in the aqueous solution, possibly due to its poor solubility), magenta - suggested ion pair resulting from the decomposition of $\mathbf{1 a}$. 


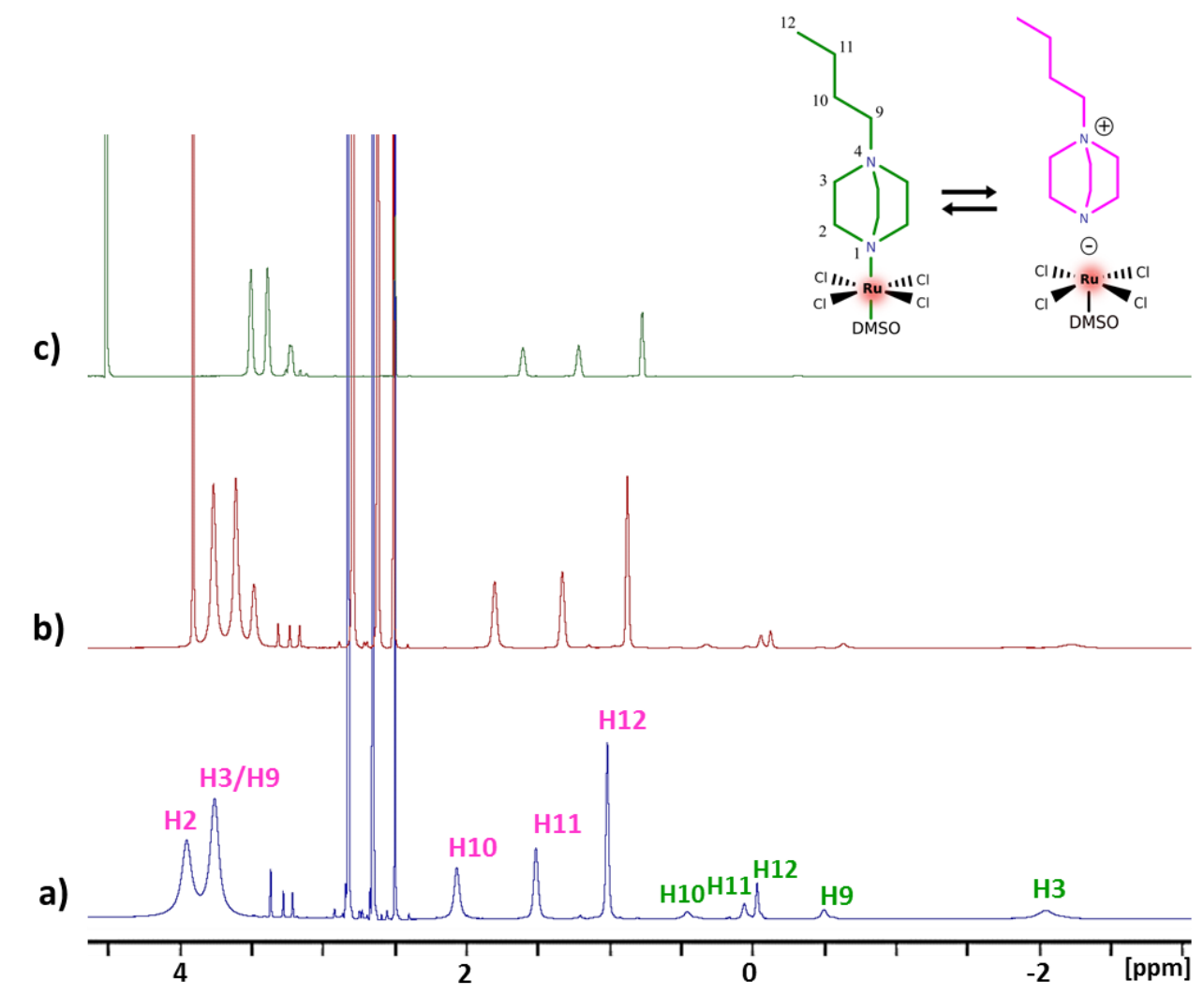

Figure S2. ${ }^{1} \mathrm{H}$ NMR spectrum of compound 1a in (a) dry DMF- $d_{7}$ (b) DMF- $d_{7}$ with a few drops of $\mathrm{D}_{2} \mathrm{O}(\mathbf{c}) \mathrm{D}_{2} \mathrm{O}$, temperature $298 \mathrm{~K}$. 


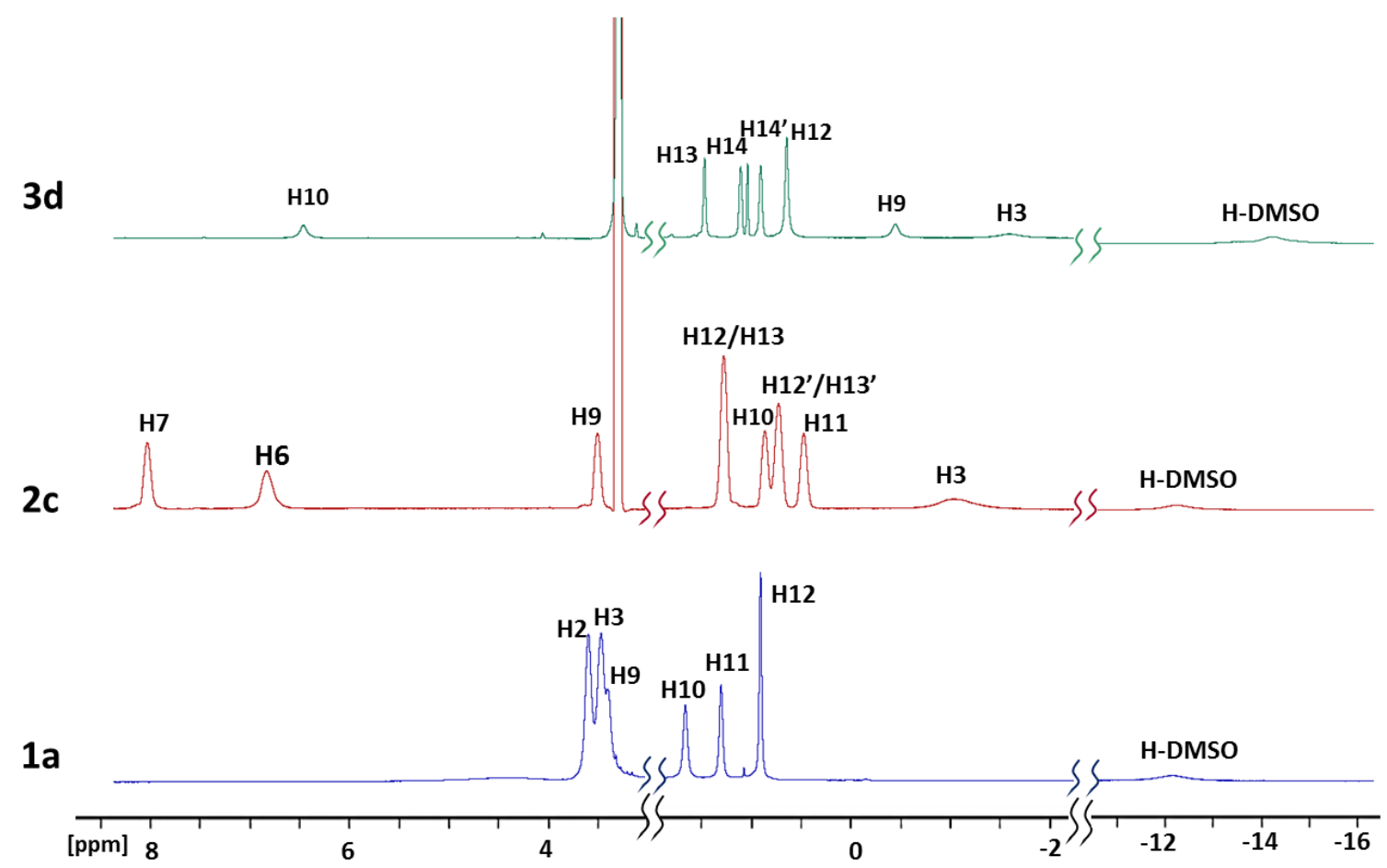

Figure S3. Portions of the ${ }^{1} \mathrm{H}$ NMR spectra of compounds 1a, 2c, and 3d in DMSO- $d_{6}$ at $298 \mathrm{~K}$. 


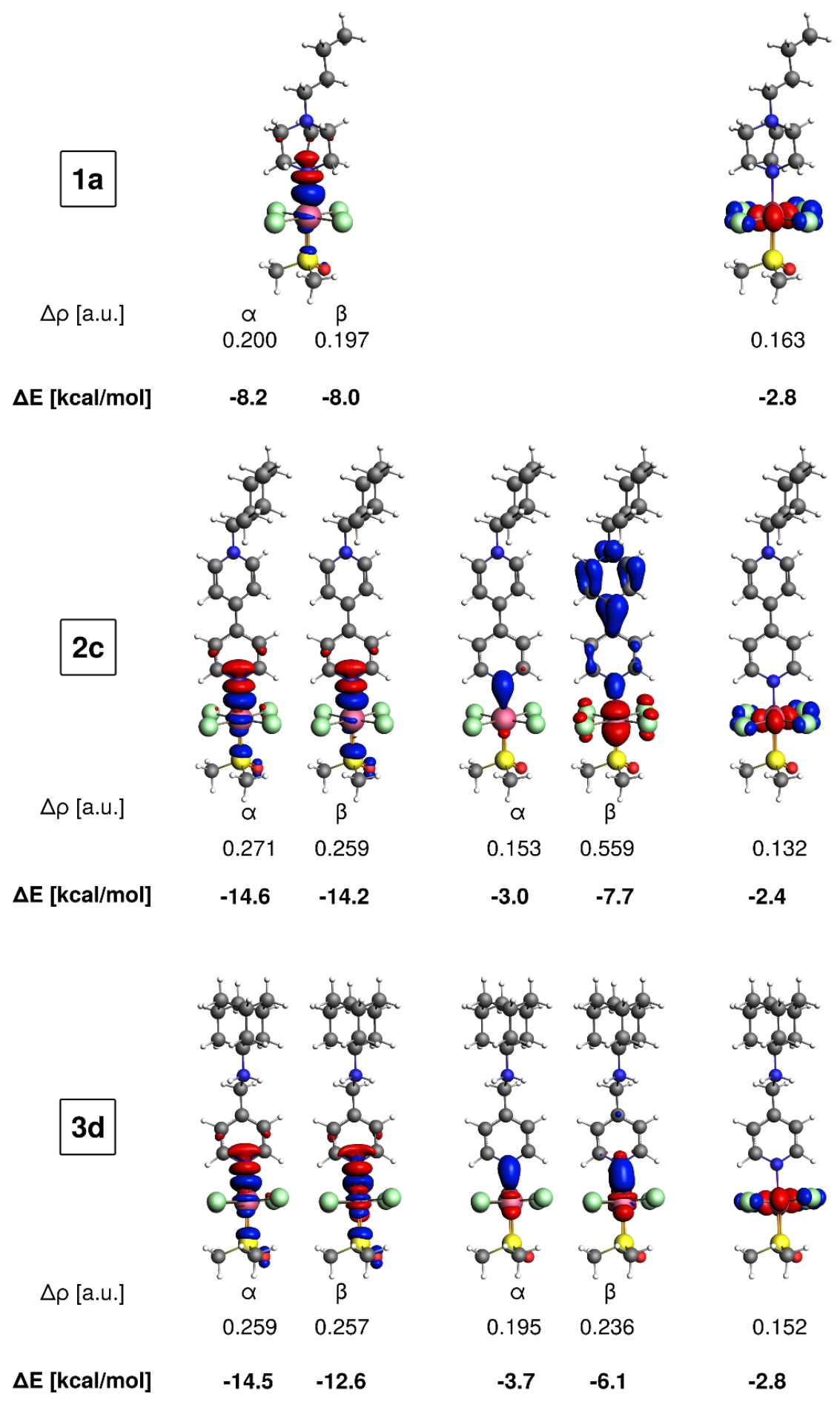

Figure S4. The NOCV channels for the formation of the $\mathrm{Ru}-\mathrm{N}$ bond in compounds $1 \mathbf{1 a}, \mathbf{2 c}$, and 3d with their corresponding contributions to the EDA orbital term $(\Delta \mathrm{E})$ and the charge transfer $(\Delta \rho)$, calculated at the ZORA level of theory (1c/PBE0/TZ2P/vacuum) and separated into $\alpha$ - and $\beta$-spin parts. The iso-surface of the electron deformation density is plotted for the value 0.001 a.u. The accumulation and depletion of electron density are shown in blue and red, respectively. 


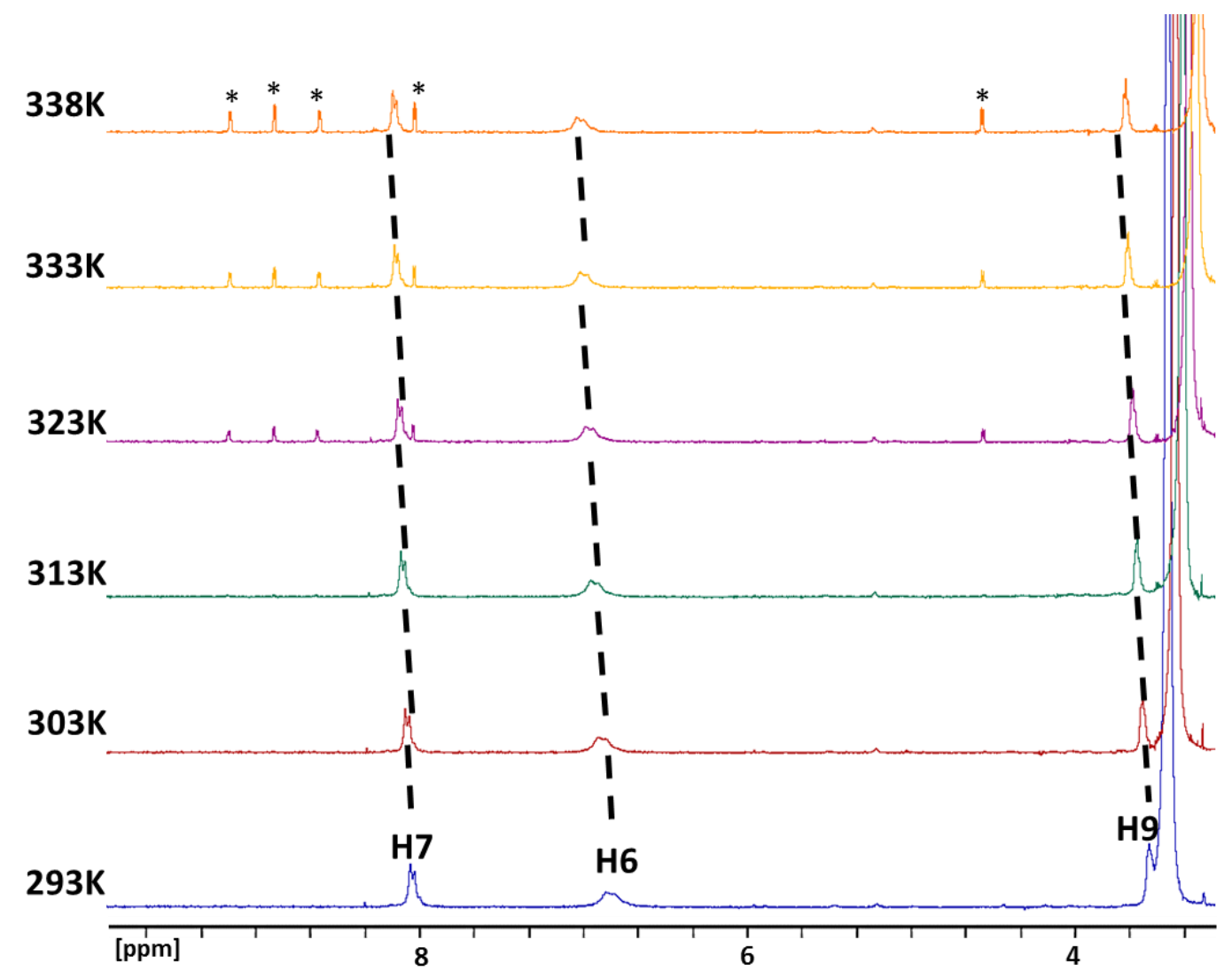

Figure S5. Portion of the ${ }^{1} \mathrm{H}$ NMR spectrum of compound $2 \mathrm{c}$ measured in DMSO- $d_{6}$ solvent at elevated temperatures (293-338 K). Note the ${ }^{1} \mathrm{H}$ NMR signals of products of decomposition $($ labeled $*)$, which appear clearly at temperatures of $323 \mathrm{~K}$ and above. 

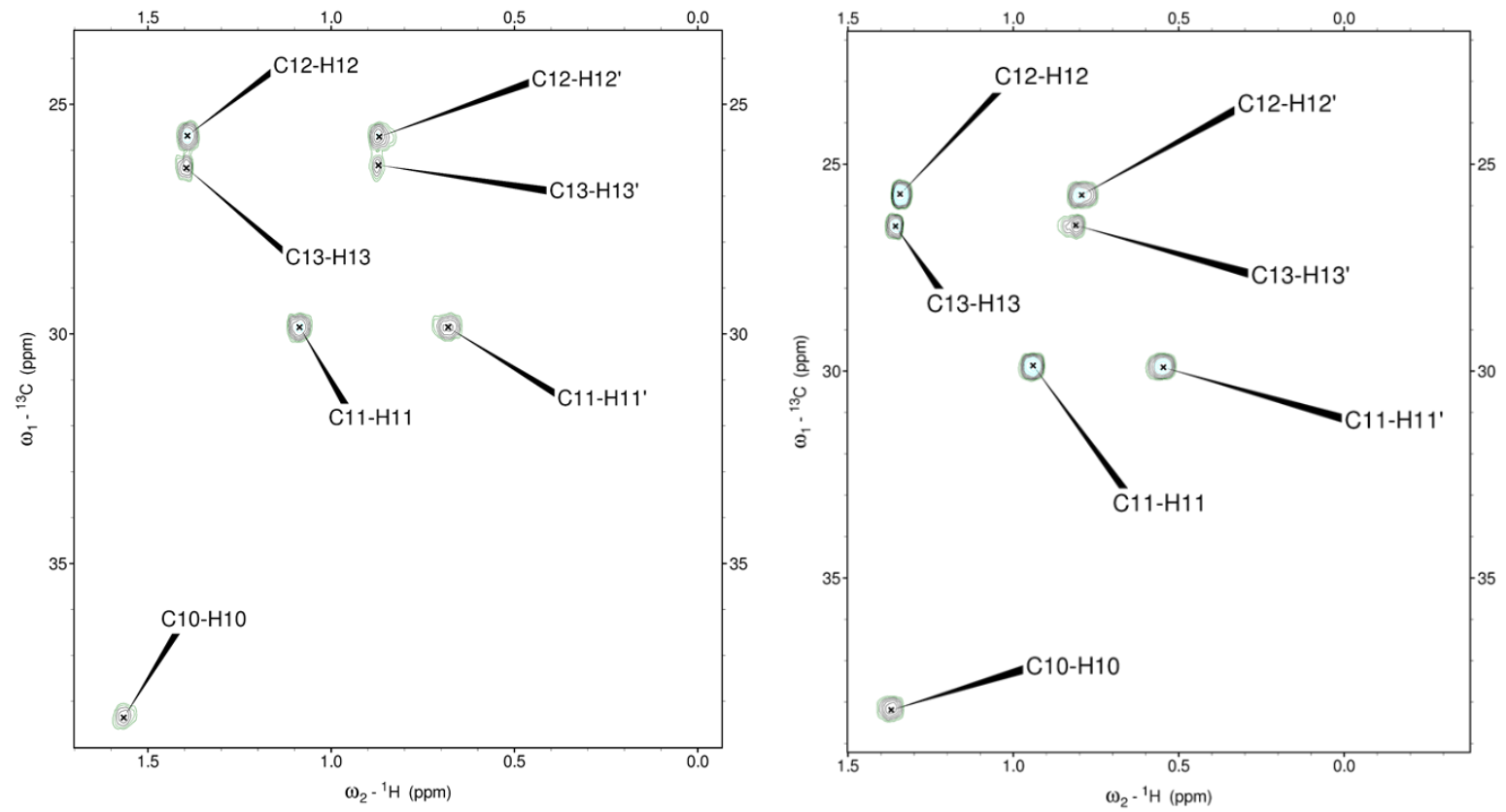

Figure S6. Portion of the ${ }^{1} \mathrm{H}_{-}{ }^{13} \mathrm{C}$ HSQC spectrum of compound $2 \mathbf{c}$ measured in DMF- $d_{7}$ (left) or DMSO- $d_{6}$ (right) at $298 \mathrm{~K}$. 


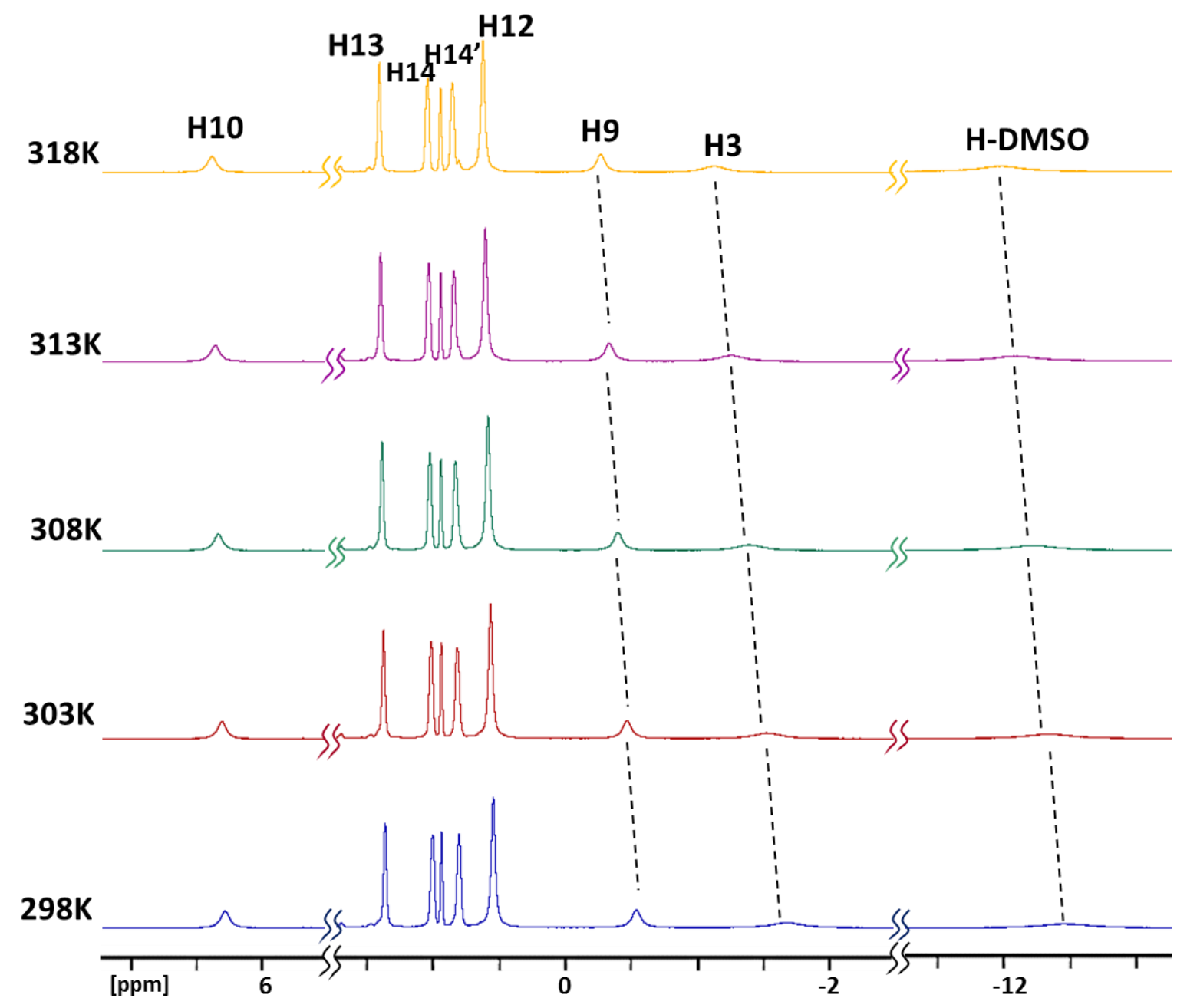

Figure S7. Portion of ${ }^{1} \mathrm{H}$ NMR spectrum of compound 3d measured in DMSO- $d_{6}$ at several elevated temperatures (298-318 K). 


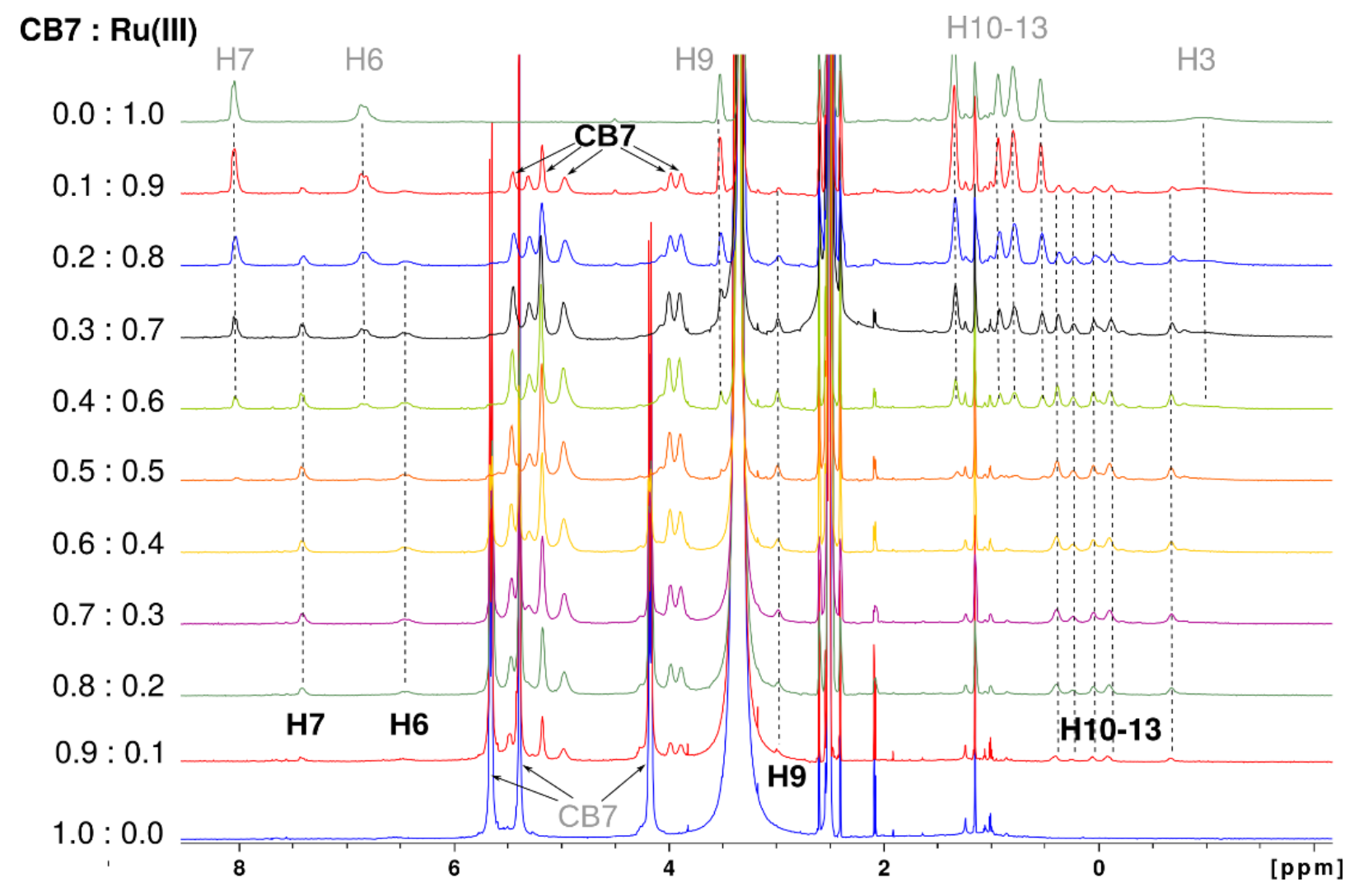

Figure S8. ${ }^{1} \mathrm{H}$ NMR spectrum of compound $2 \mathrm{c}$ in free and bound (with CB7) forms in DMSO- $d_{6}$ at $298 \mathrm{~K}$. The signals of the complex 2c@CB7 that appear upon addition of the CB7 macrocycle to a solution of $\mathbf{2 c}$ are shown in bold. 


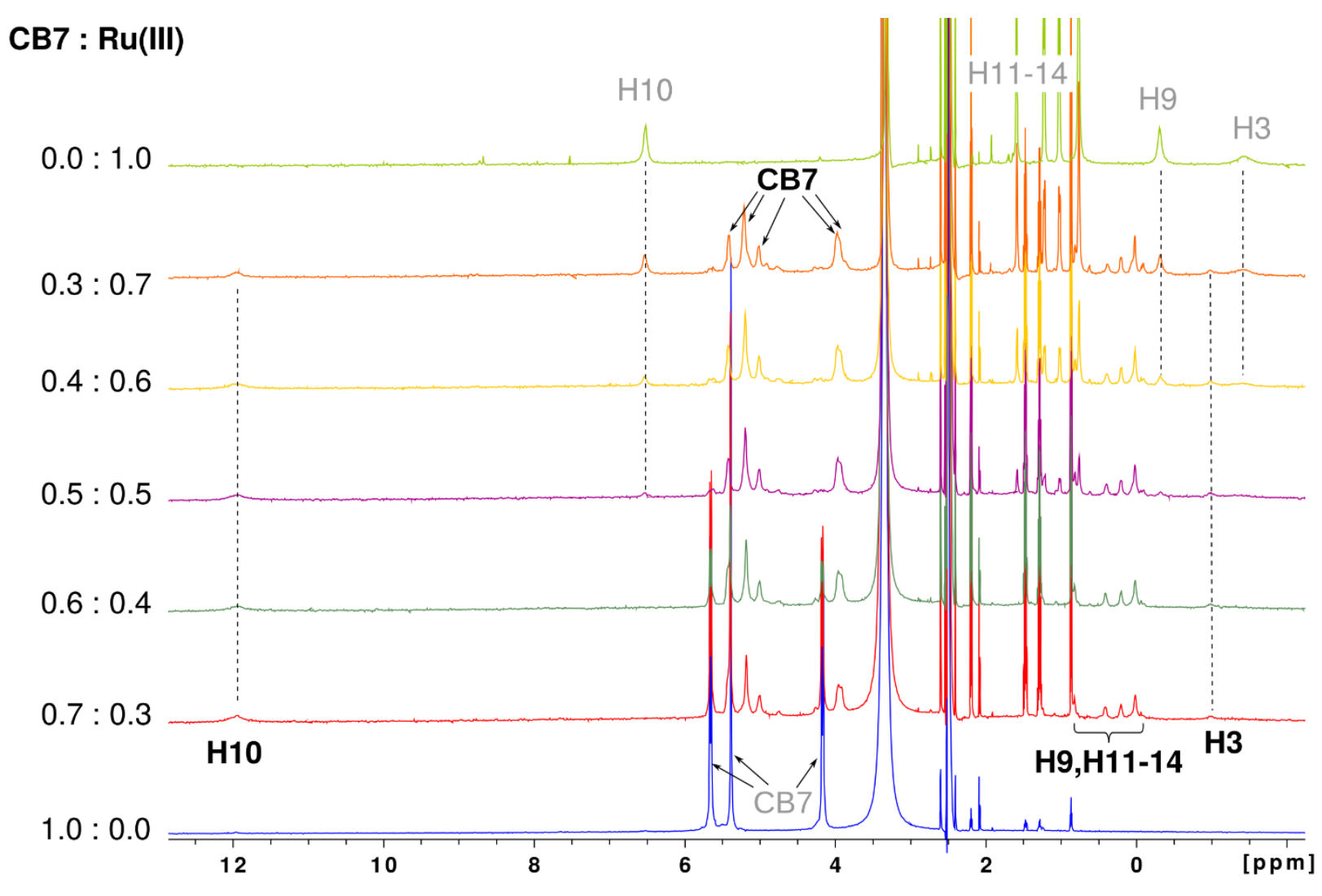

Figure S9. ${ }^{1} \mathrm{H}$ NMR spectrum of compound 3d in free and bound (with CB7) forms in DMSO- $d_{6}$ at $298 \mathrm{~K}$. The signals of the complex 3d@CB7 that appear upon addition of the CB7 macrocycle to a solution of $\mathbf{3 d}$ are shown in bold. 
The NMR spectra for systems in the slow-exchange regime (Figures S8 and S9), with the signals of the free and bound forms make it possible to calculate the binding constant directly from the relative integrals of the NMR signals. The modified Job's plots ${ }^{1}$ constructed from the NMR spectra of compounds $\mathbf{2} \mathbf{c}$ and $\mathbf{3 d}$ are shown in Figure S10 and S11, respectively.

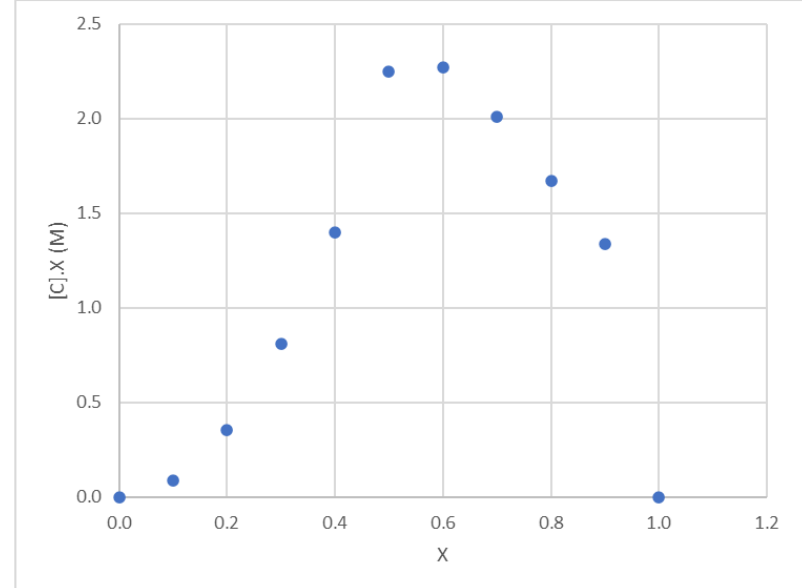

Figure S10. Modified Job's plot for the mixing of compound 2c with CB7, see Figure S8.

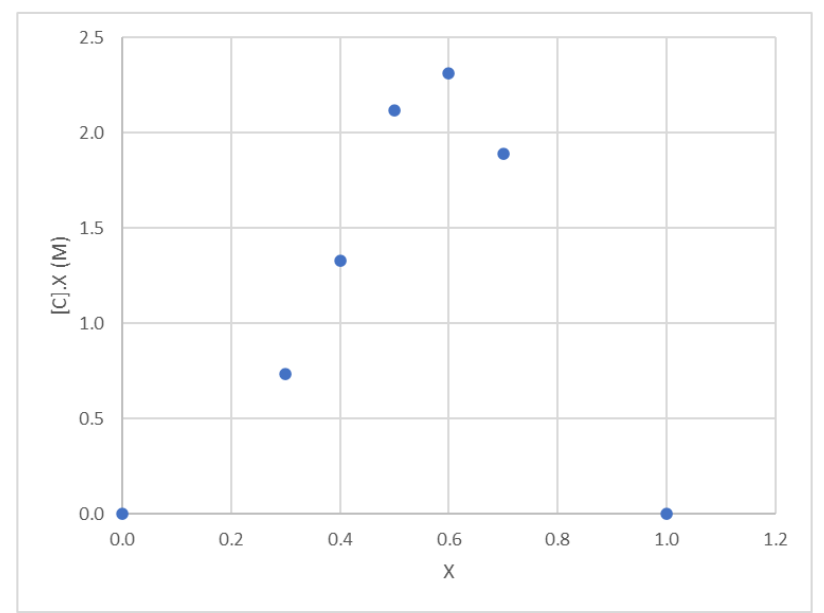

Figure S11. Modified Job's plot for the mixing of compound 3d with CB7, see Figure S9.

The binding constants ${ }^{1}$ estimated for guest compounds $2 \mathbf{c}, \mathbf{3 d}$, and some published data for several structurally related organic ligands with a CB7 host in DMSO are summarized in Table S1. 
Table S1. The binding constants for a CB7 host with guest compounds 2c, 3d, and several structurally related organic ligands ${ }^{2}$ in DMSO at room temperature.

\begin{tabular}{l|l}
\hline Compounds & Binding Constant $\left(\mathrm{M}^{-1}\right)$ in DMSO \\
\hline 2c & $(2.18 \pm 0.16) \times 10^{3}$ \\
3d & $(0.59 \pm 0.13) \times 10^{3}$ \\
Me-BiPy & $(1.3 \pm 0.5) \times 10^{3}$ \\
Hex-BiPy & $(1.9 \pm 0.04) \times 10^{3}$ \\
Bz-BiPy & \\
\hline
\end{tabular}




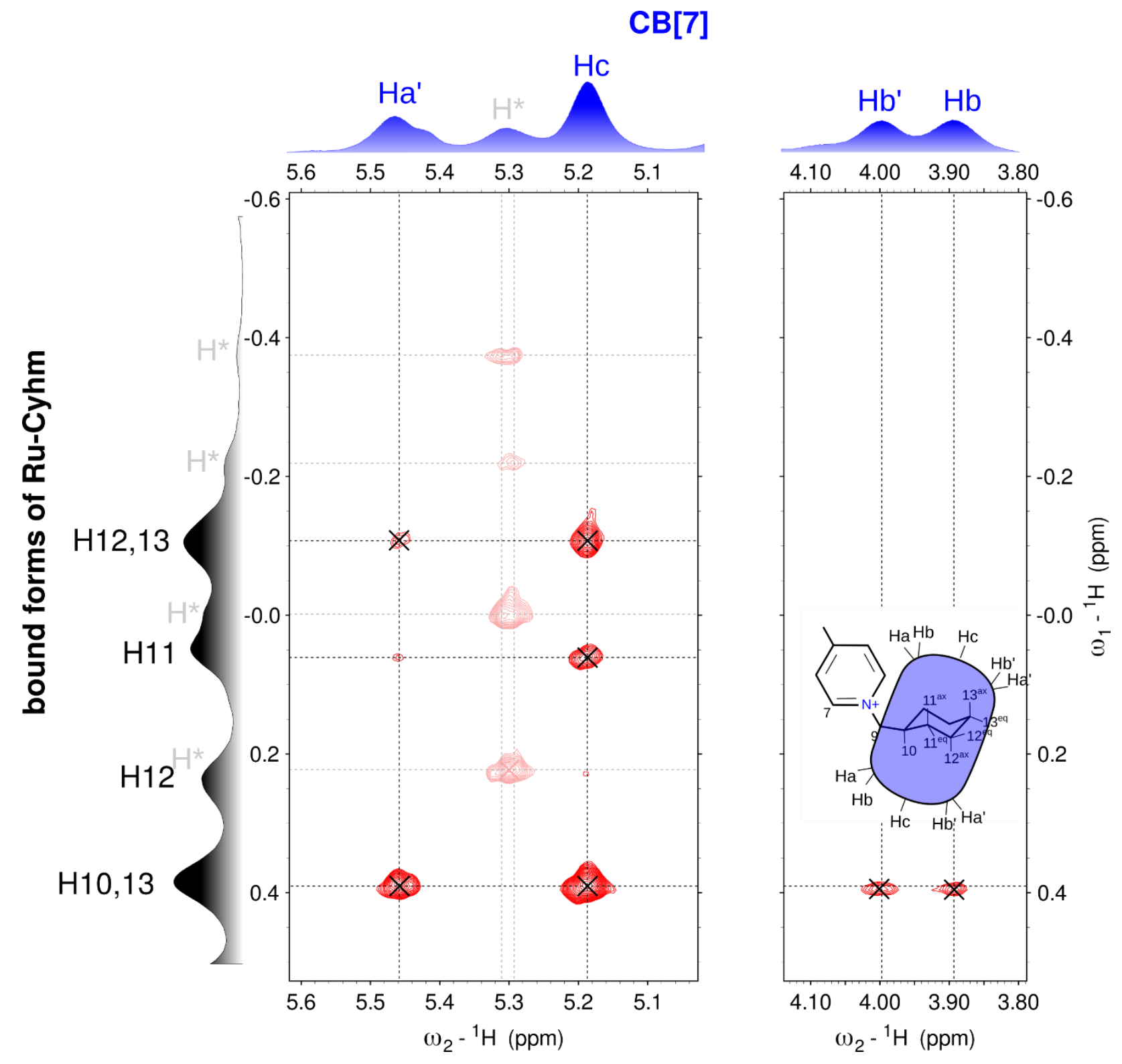

Figure S12. Portion of the $2 \mathrm{D}{ }^{1} \mathrm{H}^{1}{ }^{1} \mathrm{H}$ NOESY spectrum with cross-peaks between the cyclohexylmethyl-based (Cyhm) ligand of 2c and CB7 in DMSO- $d_{6}$ at $298 \mathrm{~K}$. The NOE contacts can result from the superposition of differently encapsulated states of the Cyhm moiety. Note that cross-peaks with $\mathrm{H}_{\mathrm{a}}$ are missing from the spectrum because of efficient paramagnetic relaxation and significant broadening of the NMR signal. The minor form of the $\mathbf{2 c} @ \mathrm{CB} 7$ complex (labeled with *, the corresponding cross-peaks are blurred) is assumed to have been produced by the solvolysis of $\mathbf{2 c}$. 

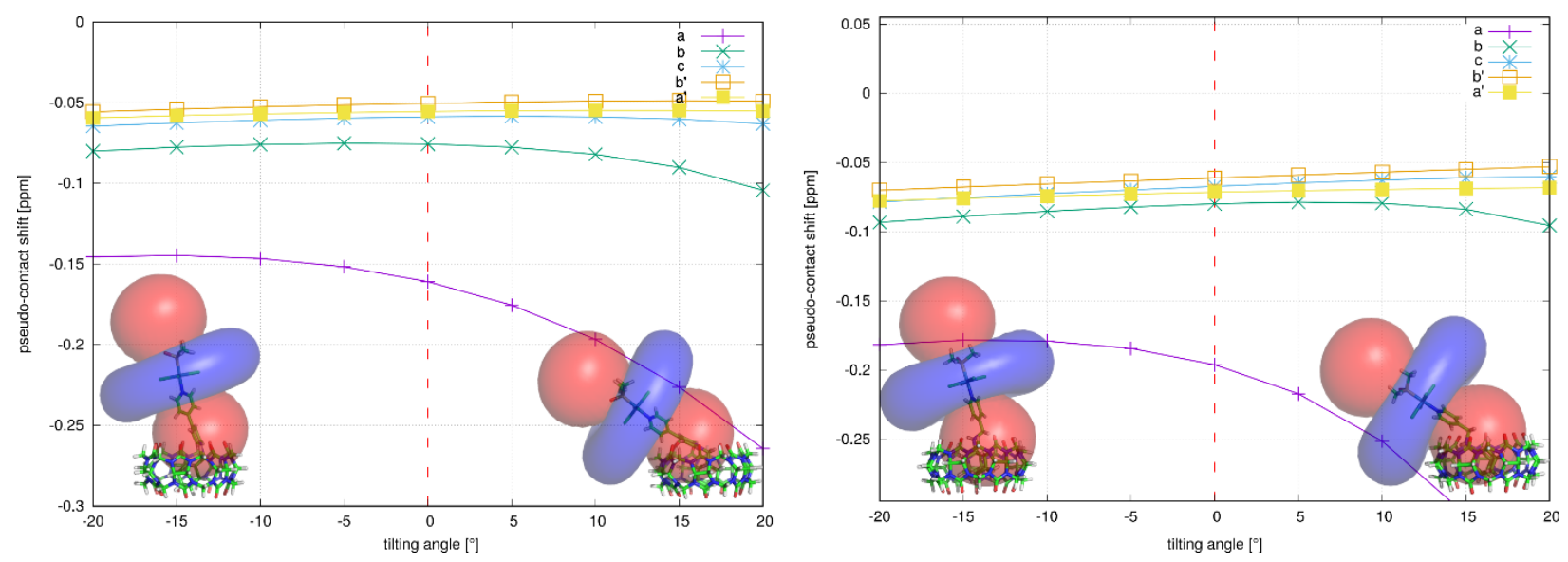

Figure S13. The effects of increased and decreased rigid tiltin of the ruthenium guest $(0$ corresponds to the optimized geometry) relative to the CB7 portal without geometry reoptimization on the $\delta^{P D}$ values (the g-tensor was not recalculated) of 2c@CB7 (left) and 3d@CB7 (right). Ha is more shielded in the more horizontal orientation of the ruthenium core (CB7 buried deeper in the [red] shielding region) and the effect is more pronounced for 3d@CB7 because the distance is smaller. Hb shows a tendency to transition, and the remaining protons of $\mathrm{CB} 7$ show a tiny increase (the small effect of the close [blue] deshielding region). The numerical SO-ZORA values for 2c@CB7 in the range $\pm 5^{\circ}$ are shown in Table $\mathrm{S} 2$ below.

Table S2. Hyperfine ${ }^{1} \mathrm{H}$ NMR shifts calculated for 2c@CB7 from the equilibrium position obtained by a rigid tilting of $\pm 5^{\circ}$. The geometry has not been re-optimized and the g-tensor has not been recalculated.

\begin{tabular}{lcccccc}
\hline $\mathbf{2 c} @ \mathrm{CB}[7]$ & \multicolumn{2}{c}{$\mathbf{- 5}$} & \multicolumn{3}{c}{$\mathbf{0}(\mathbf{e q})$} & \multicolumn{2}{c}{$\mathbf{+ 5}$} \\
\hline & $\delta_{D F T}^{H F}$ & $\delta_{D F T}^{H F a}$ & $\delta_{D F T}^{H F}$ & $\delta_{D F T}^{H F a}$ & $\delta_{D F T}^{H F}$ & $\delta_{D F T}^{H F a}$ \\
\cline { 2 - 7 } $\mathrm{Ha}$ & -0.46 & -0.28 & -0.51 & -0.31 & -0.53 & -0.33 \\
$\mathrm{Hb}$ & -0.21 & -0.14 & -0.28 & -0.18 & -0.24 & -0.16 \\
$\mathrm{Hc}$ & -0.18 & -0.11 & -0.20 & -0.13 & -0.20 & -0.13 \\
$\mathrm{Hb}^{\prime}$ & -0.16 & -0.09 & -0.16 & -0.10 & -0.16 & -0.10 \\
$\mathrm{Ha}^{\prime}$ & -0.16 & -0.09 & -0.17 & -0.10 & -0.17 & -0.10 \\
\hline
\end{tabular}


$\mathrm{X}$-ray analysis of $[\mathrm{Cyhm}-\mathrm{DABCO}-\mathrm{H}]^{+}$trans-[RuCl $\left.4(\mathrm{DMSO}-\mathrm{S})(\mathrm{ACN})\right]^{-}$obtained from the crystallization of $1 \mathrm{c}$

The significantly reduced stability of the $\mathrm{Ru}-\mathrm{N}$ bond in compounds of type $\mathbf{1}$ can be supported indirectly by observing the molecular structure shown in Figure S14, which formed during our attempts to prepare high-quality monocrystals by slowly evaporating the organic solvent from a reaction solution of $\mathbf{1} \mathbf{c}$ in acetonitrile, see Table S3.

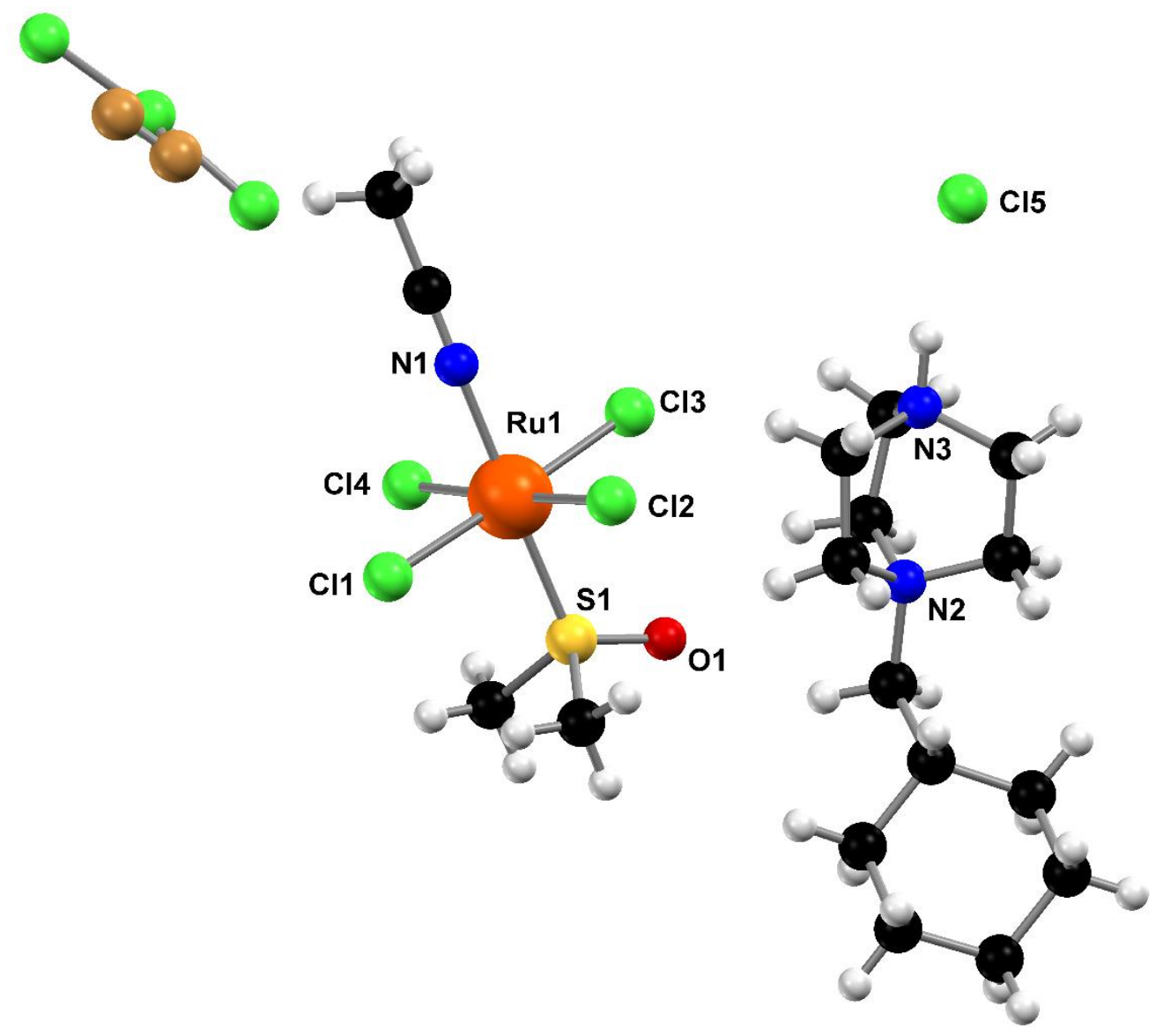

Figure S14. Molecular structure of Ru-complex $[\mathrm{Cyhm}-\mathrm{DABCO}-\mathrm{H}]^{+}$trans-[ $\mathrm{RuCl}_{4}(\mathrm{DMSO}-$ $\mathrm{S})(\mathrm{ACN})]^{-}\left(\mathrm{Br}_{0.36} \mathrm{Cl}_{0.64}\right)^{-}$obtained from the crystallization of $\mathbf{1 c}$. 
Table S3. Selected crystallographic data for the $\mathrm{Ru}(\mathrm{III})$ complex $[\mathrm{Cyhm}-\mathrm{DABCO}-\mathrm{H}]^{+}$trans- $^{-}$ $\left[\mathrm{RuCl}_{4}(\mathrm{DMSO}-\mathrm{S})(\mathrm{ACN})\right]^{-}\left(\mathrm{Br}_{0.36} \mathrm{Cl}_{0.64}\right)^{-}$formed during the crystallization of $\mathbf{1 c}$.

\begin{tabular}{|c|c|}
\hline CCDC No & 1998055 \\
\hline \multirow[t]{2}{*}{ chemical formula } & $\mathrm{C}_{17} \mathrm{H}_{35}$ \\
\hline & $\mathrm{Br}_{0.4} \mathrm{Cl}_{4.6} \mathrm{~N}_{3} \mathrm{ORuS}$ \\
\hline formula weight & 623.94 \\
\hline crystal system & Monoclinic \\
\hline space group & $C 2 / c$ \\
\hline$a(\AA)$ & $20.8698(4)$ \\
\hline$b(\AA)$ & $12.1608(2)$ \\
\hline$c(\AA)$ & $22.7784(5)$ \\
\hline$\alpha(\operatorname{deg})$ & 90 \\
\hline$\beta(\operatorname{deg})$ & $116.647(2)$ \\
\hline$\gamma(\operatorname{deg})$ & 90 \\
\hline$V\left(\AA^{3}\right)$ & $5166.99(19)$ \\
\hline$Z$ & 8 \\
\hline$D_{\text {calcd. }}\left(\mathrm{g} \mathrm{cm}^{-3}\right)$ & 1.604 \\
\hline$\mu\left(\mathrm{mm}^{-1}\right)$ & 1.741 \\
\hline measured/unique & $15549 / 4890$ \\
\hline reflections & \\
\hline data/parameters/restrai & $4890 / 278 / 19$ \\
\hline nts & \\
\hline$R_{1} / w R_{2}[\mathrm{I}>2 \sigma(\mathrm{I})]$ & $0.0424 / 0.1212$ \\
\hline$R_{1} / w R_{2}$ [all data] & $0.0463 / 0.1246$ \\
\hline GoF & 1.049 \\
\hline$\Delta \rho_{\max } / \Delta \rho_{\min }\left(\mathrm{e} \AA^{-3}\right)$ & $2.013 /-0.689$ \\
\hline
\end{tabular}




\section{References}

1. K. Hirose: A Practical Guide for the Determination of Binding Constants. J. Incl. Phenom. Macrocycl. Chem., 2001, 39, 193-209. http://dx.doi.org/10.1023/A:1011117412693.

2. A. Thangavel, A. M. M. Rawashdeh, C. Sotiriou-Leventis, N. Leventis: Simultaneous Electron Transfer from Free and Intercalated 4-Benzoylpyridinium Cations in Cucurbit[7]uril. Org. Lett., 2009, 11, 1595-1598. https://doi.org/10.1021/o19002459. 
Cartesian coordinates for DFT optimized HG complexes

\section{2c@CB7}

181

$\begin{array}{lrrr}\mathrm{Ru} & 11.37203 & 1.46436 & -1.02033 \\ \mathrm{Cl} & 11.19974 & 3.82817 & -1.23518 \\ \mathrm{Cl} & 11.61014 & 1.61005 & 1.31015 \\ \mathrm{Cl} & 11.49657 & -0.89844 & -0.78388 \\ \mathrm{Cl} & 11.08464 & 1.13996 & -3.40379 \\ \mathrm{~S} & 13.63065 & 1.65969 & -1.35095 \\ \mathrm{O} & 14.42001 & 0.41634 & -1.49369 \\ \mathrm{~N} & 9.28118 & 1.36108 & -0.69595 \\ \mathrm{~N} & 2.32152 & 1.95683 & -0.12533 \\ \mathrm{C} & 14.40105 & 2.65066 & -0.08374 \\ \mathrm{H} & 15.46244 & 2.75069 & -0.34593 \\ \mathrm{H} & 14.26256 & 2.11567 & 0.86303 \\ \mathrm{H} & 13.88599 & 3.61956 & -0.04409 \\ \mathrm{C} & 14.02810 & 2.66716 & -2.77130 \\ \mathrm{H} & 15.12231 & 2.74220 & -2.81521 \\ \mathrm{H} & 13.53801 & 3.64318 & -2.66397 \\ \mathrm{H} & 13.61821 & 2.13489 & -3.63839 \\ \mathrm{C} & 8.49019 & 0.46989 & -1.33139 \\ \mathrm{H} & 9.00616 & -0.24239 & -1.97625 \\ \mathrm{C} & 7.10973 & 0.47077 & -1.18234 \\ \mathrm{H} & 6.50858 & -0.27941 & -1.70430 \\ \mathrm{C} & 6.51016 & 1.40404 & -0.35747 \\ \mathrm{C} & 7.33635 & 2.29242 & 0.33656 \\ \mathrm{H} & 6.93066 & 3.06054 & 0.99600 \\ \mathrm{C} & 8.70101 & 2.23737 & 0.12564 \\ \mathrm{H} & 9.37285 & 2.93571 & 0.62540 \\ \mathrm{C} & 5.03895 & 1.53996 & -0.25420 \\ \mathrm{C} & 4.22802 & 1.33317 & -1.36797 \\ \mathrm{H} & 4.62310 & 0.99822 & -2.32687 \\ \mathrm{C} & 2.88056 & 1.55863 & -1.28431 \\ \mathrm{H} & 2.22577 & 1.45965 & -2.15009 \\ \mathrm{C} & 4.43142 & 1.94559 & 0.92664 \\ \mathrm{H} & 4.99579 & 2.07945 & 1.84877 \\ \mathrm{C} & 3.06873 & 2.14647 & 0.96829 \\ \mathrm{H} & 2.55574 & 2.45135 & 1.88353 \\ \mathrm{C} & 0.84075 & 2.08855 & -0.04533 \\ \mathrm{H} & 0.53716 & 2.69473 & -0.90850 \\ \mathrm{H} & 0.62153 & 2.65383 & 0.86968 \\ \mathrm{C} & 0.18223 & 0.71840 & -0.04455\end{array}$




\begin{tabular}{|c|c|c|c|c|}
\hline $\mathrm{H}$ & 0.49017 & \multicolumn{2}{|c|}{0.19193} & -0.96881 \\
\hline $\mathrm{C}$ & 0.58837 & \multicolumn{2}{|c|}{-0.13248} & 1.15041 \\
\hline $\mathrm{H}$ & 0.30873 & \multicolumn{2}{|c|}{0.40155} & 2.07837 \\
\hline $\mathrm{H}$ & 1.68201 & \multicolumn{2}{|c|}{-0.27206} & 1.18412 \\
\hline $\mathrm{C}$ & -0.11105 & \multicolumn{2}{|c|}{-1.49162} & 1.12499 \\
\hline $\mathrm{H}$ & 0.23899 & \multicolumn{2}{|c|}{-2.06769} & 0.25038 \\
\hline $\mathrm{H}$ & 0.18312 & \multicolumn{2}{|c|}{-2.07159} & 2.01201 \\
\hline $\mathrm{C}$ & -1.61237 & \multicolumn{2}{|c|}{-1.33397} & 1.06520 \\
\hline $\mathrm{H}$ & -1.98146 & \multicolumn{2}{|c|}{-0.87921} & 1.99988 \\
\hline $\mathrm{H}$ & -2.07905 & \multicolumn{2}{|c|}{-2.32979} & 1.00246 \\
\hline $\mathrm{C}$ & -2.03497 & \multicolumn{2}{|c|}{-0.47185} & -0.11479 \\
\hline $\mathrm{H}$ & -1.80009 & \multicolumn{2}{|c|}{-0.99316} & -1.06068 \\
\hline $\mathrm{H}$ & -3.12380 & \multicolumn{2}{|c|}{-0.30844} & -0.11097 \\
\hline $\mathrm{C}$ & -1.34233 & \multicolumn{2}{|c|}{0.87546} & -0.09191 \\
\hline $\mathrm{H}$ & -1.66613 & \multicolumn{2}{|c|}{1.44829} & 0.79631 \\
\hline $\mathrm{H}$ & -1.63231 & \multicolumn{2}{|c|}{1.47813} & -0.9693 \\
\hline $\mathrm{O}$ & 4.47452 & -1.31869 & 1.3 & \\
\hline $\mathrm{O}$ & -0.23360 & -4.99212 & 2.3 & \\
\hline $\mathrm{O}$ & 4.79294 & -1.83652 & -2.2 & \\
\hline $\mathrm{O}$ & -0.60279 & -4.53849 & -1.2 & \\
\hline $\mathrm{O}$ & 3.02497 & 0.52571 & -4.2 & \\
\hline $\mathrm{O}$ & -2.29243 & -2.30086 & -3.8 & \\
\hline $\mathrm{O}$ & 1.01316 & 3.44158 & -3.1 & \\
\hline $\mathrm{O}$ & -4.28917 & 0.59626 & -3.5 & \\
\hline $\mathrm{O}$ & 0.24834 & 5.30296 & -0.1 & \\
\hline $\mathrm{O}$ & -4.86373 & 1.87775 & -0.1 & \\
\hline $\mathrm{O}$ & 1.15437 & 4.04819 & 3.20 & \\
\hline $\mathrm{O}$ & -3.83548 & 0.51610 & 3.2 & \\
\hline $\mathrm{O}$ & 3.09034 & 1.26659 & 3.8 & \\
\hline $\mathrm{O}$ & -1.68160 & -2.58883 & 4.4 & \\
\hline $\mathrm{N}$ & 3.89651 & -3.54393 & & \\
\hline $\mathrm{N}$ & 1.87269 & -4.86632 & 1.4 & \\
\hline $\mathrm{N}$ & 1.59235 & -4.13194 & 3.5 & \\
\hline $\mathrm{N}$ & 3.34628 & -2.49584 & 3.0 & \\
\hline $\mathrm{N}$ & 3.10553 & -3.00582 & -3.2 & \\
\hline $\mathrm{N}$ & 0.96817 & -4.09259 & -2.9 & \\
\hline $\mathrm{N}$ & 1.67110 & -4.84280 & -0.9 & \\
\hline $\mathrm{N}$ & 3.79934 & -3.70505 & -1.3 & \\
\hline $\mathrm{N}$ & 0.94446 & 0.36606 & -5.2 & \\
\hline $\mathrm{N}$ & -1.19627 & -0.78077 & -5.2 & 36 \\
\hline $\mathrm{N}$ & -0.08083 & -2.56281 & -4.4 & \\
\hline $\mathrm{N}$ & 2.07257 & -1.49638 & -4.8 & \\
\hline $\mathrm{N}$ & -1.23343 & 3.93722 & -3.3 & \\
\hline $\mathrm{N}$ & -3.32439 & 2.69541 & -3.4 & \\
\hline $\mathrm{N}$ & -2.52086 & 1.23325 & -4.8 & \\
\hline $\mathrm{N}$ & -0.34806 & 2.31246 & -4.6 & \\
\hline
\end{tabular}




$\begin{array}{lrrr}\mathrm{N} & -1.75643 & 5.01331 & 1.00179 \\ \mathrm{~N} & -3.72534 & 3.55571 & 1.01189 \\ \mathrm{~N} & -3.79300 & 3.60061 & -1.20995 \\ \mathrm{~N} & -1.73870 & 4.90670 & -1.21425 \\ \mathrm{~N} & -0.16953 & 2.83327 & 4.70167 \\ \mathrm{~N} & -2.06831 & 1.29765 & 4.49312 \\ \mathrm{~N} & -3.20678 & 2.73396 & 3.22940 \\ \mathrm{~N} & -1.15622 & 4.06452 & 3.13272 \\ \mathrm{~N} & 2.40549 & -0.84657 & 4.49825 \\ \mathrm{~N} & 0.57401 & -2.40947 & 4.87895 \\ \mathrm{~N} & -0.71301 & -0.61298 & 5.11222 \\ \mathrm{~N} & 1.20045 & 0.89245 & 5.14030 \\ \mathrm{C} & 3.94662 & -2.33687 & 1.76355 \\ \mathrm{C} & 3.23331 & -4.56302 & 1.88641 \\ \mathrm{H} & 3.84388 & -5.47990 & 1.93098 \\ \mathrm{C} & 0.96171 & -4.68610 & 2.44601 \\ \mathrm{C} & 2.99564 & -3.88507 & 3.26182 \\ \mathrm{H} & 3.61413 & -4.29340 & 4.07830 \\ \mathrm{C} & 4.61260 & -3.79964 & -0.11341 \\ \mathrm{H} & 5.39507 & -3.03411 & -0.21137 \\ \mathrm{H} & 5.06855 & -4.80082 & -0.05129 \\ \mathrm{C} & 1.57623 & -5.62614 & 0.25772 \\ \mathrm{H} & 2.25996 & -6.48811 & 0.18355 \\ \mathrm{H} & 0.53894 & -5.97189 & 0.36115 \\ \mathrm{C} & 3.97534 & -2.75079 & -2.25509 \\ \mathrm{C} & 2.39028 & -4.25583 & -3.10719 \\ \mathrm{H} & 2.60369 & -4.94364 & -3.94250 \\ \mathrm{C} & 0.55737 & -4.49211 & -1.67200 \\ \mathrm{C} & 2.88679 & -4.75953 & -1.72599 \\ \mathrm{H} & 3.40271 & -5.73336 & -1.75393 \\ \mathrm{C} & 3.20125 & -2.34152 & -4.55655 \\ \mathrm{H} & 4.09247 & -1.70066 & -4.51861 \\ \mathrm{H} & 3.31125 & -3.09211 & -5.35599 \\ \mathrm{C} & 0.05112 & -3.93343 & -4.02779 \\ \mathrm{H} & -0.94045 & -4.25428 & -3.68143 \\ \mathrm{H} & 0.38605 & -4.56500 & -4.86781 \\ \mathrm{C} & 2.11040 & -0.12960 & -4.73069 \\ \mathrm{C} & 0.09752 & -0.65410 & -5.82384 \\ \mathrm{H} & -0.00624 & -0.50765 & -6.91199 \\ \mathrm{C} & -1.29127 & -1.92032 & -4.44954 \\ \mathrm{C} & 0.83436 & -1.96542 & -5.42902 \\ \mathrm{H} & 1.02300 & -2.64928 & -6.27331 \\ \mathrm{C} & 0.72367 & 1.77763 & -5.44539 \\ \mathrm{H} & 0.48397 & 1.97480 & -6.50331 \\ \mathrm{H} & 1.65388 & 2.28996 & -5.16707 \\ \mathrm{C} & -2.35389 & -0.01122 & -5.59990\end{array}$




\begin{tabular}{|c|c|c|c|}
\hline & 3.24614 & -0.61611 & \\
\hline & -2.28488 & 0.20640 & \\
\hline & -0.07254 & 3.25003 & \\
\hline & -2.35023 & 3.46798 & \\
\hline & -2.83392 & 4.30759 & \\
\hline & -3.45191 & 1.41757 & \\
\hline & -1.71125 & 2.41218 & \\
\hline & -1.72393 & 2.69585 & \\
\hline & -1.25325 & 5.12534 & \\
\hline & -1.87203 & 5.88732 & \\
\hline & -0.2 & 5.48646 & \\
\hline & -4.29713 & 3.28794 & -2.5 \\
\hline & -4.6 & 4.20831 & \\
\hline & -5.09989 & 2.5 & -2 . \\
\hline & -0.95233 & 5.0 & \\
\hline & -3.1 & & \\
\hline & -3.75316 & 5.6 & \\
\hline & -4.1 & 2.8 & \\
\hline & -3.1 & 12 & -0 . \\
\hline & -3.6 & 27 & \\
\hline & -1.2 & 5.2 & \\
\hline & -1.9 & 34 & \\
\hline & -0.2 & 5.7 & \\
\hline & -4.2 & 3.2 & \\
\hline & -4.6 & & \\
\hline & -4.98 & 52 & \\
\hline & 0.04 & 19 & \\
\hline & -1.57934 & 62 & \\
\hline & -1.8 & 79 & \\
\hline & -3.10749 & & \\
\hline & -2.26440 & 3.5 & \\
\hline & $-2.7 \varepsilon$ & & \\
\hline & 0.90137 & 2.26 & \\
\hline$H$ & 0.6 & 08 & \\
\hline & 1.79893 & & \\
\hline & -1.95085 & 93 & \\
\hline & -2.05673 & 0.38232 & \\
\hline 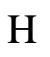 & -2.76321 & -0.57391 & \\
\hline & 2.31225 & 0.51811 & 4.4 \\
\hline 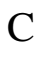 & 1.45958 & -1.42134 & 5.4 \\
\hline 1 & 1.98381 & -1.82575 & \\
\hline 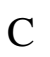 & -0.70861 & -1.94533 & \\
\hline 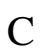 & 0.53033 & -0.22950 & \\
\hline & 0.37874 & -0.04685 & \\
\hline$\Omega$ & 3.59660 & -1.54649 & 4.0 \\
\hline$-\gamma$ & 4.28825 & -0.78797 & 3.67 \\
\hline
\end{tabular}




$\begin{array}{cccc}\mathrm{H} & 4.05176 & -2.07044 & 4.92253 \\ \mathrm{C} & 0.91955 & -3.80730 & 4.75489 \\ \mathrm{H} & -0.01636 & -4.38104 & 4.76986 \\ \mathrm{H} & 1.55830 & -4.09964 & 5.60233\end{array}$

\section{3d@CB7}

182

$\begin{array}{lccr}\mathrm{Ru} & -2.80289 & 9.73819 & 1.12516 \\ \mathrm{~S} & -3.75261 & 11.78346 & 1.47027 \\ \mathrm{C} & -2.51710 & 13.00769 & 1.83493 \\ \mathrm{Cl} & -3.90338 & 9.45602 & -0.91817 \\ \mathrm{Cl} & -0.98352 & 10.72690 & 0.00003 \\ \mathrm{Cl} & -1.65316 & 9.95107 & 3.17947 \\ \mathrm{Cl} & -4.57389 & 8.64621 & 2.20982 \\ \mathrm{~N} & -1.89633 & 7.85858 & 0.83872 \\ \mathrm{C} & -1.38369 & 7.51609 & -0.34491 \\ \mathrm{C} & -0.75701 & 6.30234 & -0.55165 \\ \mathrm{C} & -0.64199 & 5.40766 & 0.50006 \\ \mathrm{C} & -1.18365 & 5.76741 & 1.72688 \\ \mathrm{C} & -1.80512 & 6.99202 & 1.85229 \\ \mathrm{C} & -4.64455 & 11.82008 & 3.00642 \\ \mathrm{O} & -4.63544 & 12.35614 & 0.43658 \\ \mathrm{H} & -3.03823 & 13.92709 & 2.09878 \\ \mathrm{H} & -1.91819 & 13.13228 & 0.93663 \\ \mathrm{H} & -1.89635 & 12.64210 & 2.65060 \\ \mathrm{H} & -5.49791 & 11.15629 & 2.89716 \\ \mathrm{H} & -4.95718 & 12.84910 & 3.17913 \\ \mathrm{H} & -3.98793 & 11.45672 & 3.79507 \\ \mathrm{H} & -1.14117 & 5.10194 & 2.58032 \\ \mathrm{H} & -2.24526 & 7.30701 & 2.78595 \\ \mathrm{H} & -0.36527 & 6.06646 & -1.53280 \\ \mathrm{C} & 0.04102 & 4.08744 & 0.31506 \\ \mathrm{H} & -1.48856 & 8.24069 & -1.13779 \\ \mathrm{~N} & -0.93840 & 2.98204 & 0.47312 \\ \mathrm{C} & -0.42955 & 1.58002 & 0.22900 \\ \mathrm{C} & -0.14917 & 1.38511 & -1.25586 \\ \mathrm{C} & 0.83501 & 1.30878 & 1.03257 \\ \mathrm{C} & -1.54135 & 0.63514 & 0.67853 \\ \mathrm{H} & -1.04538 & 1.61256 & -1.83599 \\ \mathrm{C} & 0.28338 & -0.06272 & -1.49092 \\ \mathrm{H} & 0.63884 & 2.06097 & -1.59669 \\ \mathrm{C} & 1.26527 & -0.14086 & 0.79762\end{array}$




\begin{tabular}{|c|c|c|c|c|}
\hline $\mathrm{H}$ & 0.65142 & \multicolumn{2}{|c|}{1.48903} & 2.09371 \\
\hline $\mathrm{H}$ & 1.64301 & \multicolumn{2}{|c|}{1.97510} & 0.72281 \\
\hline $\mathrm{H}$ & -1.33850 & \multicolumn{2}{|c|}{3.03826} & 1.41782 \\
\hline $\mathrm{C}$ & -1.10834 & \multicolumn{2}{|c|}{-0.81001} & 0.43927 \\
\hline $\mathrm{H}$ & -2.46243 & \multicolumn{2}{|c|}{0.84954} & 0.12754 \\
\hline $\mathrm{H}$ & -1.75039 & \multicolumn{2}{|c|}{0.79147} & 1.74262 \\
\hline $\mathrm{C}$ & -0.83134 & \multicolumn{2}{|c|}{-1.00673} & -1.04940 \\
\hline $\mathrm{H}$ & -1.73792 & \multicolumn{2}{|c|}{-0.81354} & -1.62985 \\
\hline $\mathrm{H}$ & -0.54321 & \multicolumn{2}{|c|}{-2.04166} & -1.24879 \\
\hline $\mathrm{C}$ & 1.54875 & \multicolumn{2}{|c|}{-0.35151} & -0.68769 \\
\hline $\mathrm{H}$ & 1.87247 & \multicolumn{2}{|c|}{-1.38110} & -0.86220 \\
\hline $\mathrm{H}$ & 2.36032 & \multicolumn{2}{|c|}{0.30489} & -1.01428 \\
\hline $\mathrm{H}$ & 0.48382 & \multicolumn{2}{|c|}{-0.19736} & -2.55616 \\
\hline $\mathrm{C}$ & 0.15691 & \multicolumn{2}{|c|}{-1.08771} & 1.24733 \\
\hline $\mathrm{H}$ & -1.91219 & \multicolumn{2}{|c|}{-1.47596} & 0.76092 \\
\hline $\mathrm{H}$ & 0.47531 & \multicolumn{2}{|c|}{-2.12414} & 1.11184 \\
\hline $\mathrm{H}$ & -0.03995 & \multicolumn{2}{|c|}{-0.95384} & 2.31478 \\
\hline $\mathrm{H}$ & 2.17341 & \multicolumn{2}{|c|}{-0.33009} & 1.37381 \\
\hline $\mathrm{H}$ & -1.73188 & \multicolumn{2}{|c|}{3.15642} & -0.16929 \\
\hline $\mathrm{H}$ & 0.47494 & \multicolumn{2}{|c|}{4.01141} & -0.67930 \\
\hline $\mathrm{H}$ & 0.82408 & \multicolumn{2}{|c|}{3.94740} & 1.05878 \\
\hline $\mathrm{H}$ & 6.29579 & 0.62138 & -2.89976 & \\
\hline $\mathrm{H}$ & 6.77635 & -1.05870 & -1.13661 & \\
\hline $\mathrm{H}$ & 5.22108 & -0.88097 & -4.52064 & \\
\hline $\mathrm{H}$ & 5.30561 & 2.00797 & -2.37242 & \\
\hline $\mathrm{C}$ & 5.34859 & 0.92187 & -2.44058 & \\
\hline $\mathrm{H}$ & 5.81596 & -2.57805 & -2.81787 & \\
\hline $\mathrm{C}$ & 5.77556 & -0.89158 & -0.72800 & \\
\hline $\mathrm{H}$ & 6.70965 & -1.01225 & 1.28986 & \\
\hline $\mathrm{C}$ & 4.26231 & -0.73411 & -4.01493 & \\
\hline $\mathrm{N}$ & 5.29507 & 0.40981 & -1.10400 & \\
\hline $\mathrm{N}$ & 4.26809 & 0.50479 & -3.28474 & \\
\hline $\mathrm{H}$ & 3.33829 & -0.66723 & -6.03962 & \\
\hline $\mathrm{C}$ & 4.81981 & -2.56904 & -2.36524 & \\
\hline $\mathrm{C}$ & 5.04556 & 1.21619 & -0.02454 & \\
\hline $\mathrm{C}$ & 5.73459 & -0.85224 & 0.82047 & \\
\hline $\mathrm{C}$ & 3.06572 & -0.57104 & -4.98471 & \\
\hline $\mathrm{C}$ & 3.32074 & 1.38862 & -3.73513 & \\
\hline $\mathrm{O}$ & 4.72707 & 2.38577 & -0.06187 & \\
\hline $\mathrm{N}$ & 3.91619 & -1.90160 & -3.25418 & \\
\hline $\mathrm{N}$ & 4.91327 & -1.99324 & -1.05565 & \\
\hline $\mathrm{H}$ & 4.46550 & -3.59188 & -2.24730 & \\
\hline $\mathrm{H}$ & 6.18436 & 0.77085 & 2.92872 & \\
\hline $\mathrm{O}$ & 3.17399 & 2.53299 & -3.36225 & \\
\hline $\mathrm{N}$ & 5.25423 & 0.47064 & 1.10684 & \\
\hline $\mathrm{H}$ & 1.83067 & 1.21415 & -6.57224 & \\
\hline
\end{tabular}




\begin{tabular}{|c|c|c|c|}
\hline $\mathrm{N}$ & 2.59336 & 0.75591 & -4.70956 \\
\hline $\mathrm{H}$ & 5.23502 & 2.13651 & 2.28535 \\
\hline $\mathrm{C}$ & 5.26170 & 1.05550 & 2.41400 \\
\hline $\mathrm{H}$ & 1.77184 & 2.51248 & -5.35183 \\
\hline $\mathrm{C}$ & 1.63565 & 1.44545 & -5.52100 \\
\hline $\mathrm{N}$ & 4.83759 & -1.92415 & 1.15591 \\
\hline $\mathrm{N}$ & 2.17916 & -1.63624 & -4.59905 \\
\hline $\mathrm{C}$ & 2.71364 & -2.44190 & -3.62615 \\
\hline $\mathrm{C}$ & 4.41029 & -2.61979 & 0.05418 \\
\hline $\mathrm{H}$ & 5.64494 & -2.43522 & 2.98840 \\
\hline $\mathrm{H}$ & 1.28010 & -1.95109 & -6.43247 \\
\hline $\mathrm{C}$ & 4.67279 & -2.43204 & 2.48504 \\
\hline $\mathrm{C}$ & 1.03535 & -2.02758 & -5.36800 \\
\hline $\mathrm{O}$ & 2.22508 & -3.46647 & -3.19871 \\
\hline $\mathrm{O}$ & 3.73578 & -3.62781 & 0.06354 \\
\hline $\mathrm{H}$ & 5.00081 & -0.65192 & 4.56844 \\
\hline $\mathrm{H}$ & 4.30313 & -3.45295 & 2.40154 \\
\hline $\mathrm{N}$ & 4.14126 & 0.69725 & 3.23308 \\
\hline $\mathrm{H}$ & 0.81200 & -3.06420 & -5.12023 \\
\hline $\mathrm{N}$ & 0.26803 & 1.13795 & -5.22391 \\
\hline $\mathrm{H}$ & -0.26934 & -0.08254 & -6.82459 \\
\hline $\mathrm{C}$ & 4.07040 & -0.50809 & 4.01141 \\
\hline $\mathrm{N}$ & 3.73326 & -1.70398 & 3.28725 \\
\hline $\mathrm{C}$ & -0.43033 & -0.00282 & -5.74560 \\
\hline $\mathrm{O}$ & 3.04557 & 2.72323 & 3.12440 \\
\hline $\mathrm{N}$ & -0.14801 & -1.26056 & -5.10539 \\
\hline $\mathrm{C}$ & 3.15856 & 1.59503 & 3.55534 \\
\hline $\mathrm{O}$ & -0.25255 & 3.11402 & -4.15515 \\
\hline $\mathrm{C}$ & -0.56216 & 2.02862 & -4.59744 \\
\hline $\mathrm{C}$ & 2.83227 & -0.28148 & 4.91407 \\
\hline $\mathrm{C}$ & 2.55592 & -2.26417 & 3.71129 \\
\hline $\mathrm{H}$ & 3.06630 & -0.25421 & 5.98250 \\
\hline $\mathrm{N}$ & 2.33860 & 0.99727 & 4.47850 \\
\hline $\mathrm{C}$ & -1.26239 & -1.82218 & -4.53594 \\
\hline $\mathrm{C}$ & -1.90411 & 0.26815 & -5.35549 \\
\hline $\mathrm{N}$ & -1.82132 & 1.48049 & -4.58480 \\
\hline $\mathrm{H}$ & -2.56653 & 0.40727 & -6.21509 \\
\hline $\mathrm{O}$ & 2.11076 & -3.33884 & 3.37000 \\
\hline $\mathrm{O}$ & -1.34193 & -2.93749 & -4.06740 \\
\hline $\mathrm{N}$ & 1.98756 & -1.40602 & 4.61990 \\
\hline $\mathrm{H}$ & 1.40335 & 2.76866 & 4.84962 \\
\hline $\mathrm{N}$ & -2.28216 & -0.90773 & -4.62285 \\
\hline $\mathrm{H}$ & -2.60572 & 3.25024 & -3.95707 \\
\hline $\mathrm{C}$ & 1.33735 & 1.73433 & 5.18395 \\
\hline $\mathrm{H}$ & 1.53867 & 1.68471 & 6.25939 \\
\hline $\mathrm{C}$ & -2.96443 & 2.25403 & -4.21301 \\
\hline
\end{tabular}




$\begin{array}{lrrr}\mathrm{H} & -3.65648 & 2.31951 & -5.05915 \\ \mathrm{C} & 0.88888 & -1.80775 & 5.45111 \\ \mathrm{H} & 1.17002 & -1.67414 & 6.50051 \\ \mathrm{H} & 0.70242 & -2.86271 & 5.25627 \\ \mathrm{O} & -0.70635 & 3.07916 & 3.67984 \\ \mathrm{~N} & -0.00733 & 1.28547 & 4.95201 \\ \mathrm{C} & -3.64326 & -1.26096 & -4.34002 \\ \mathrm{H} & -4.26109 & -1.02189 & -5.21131 \\ \mathrm{H} & -3.66966 & -2.33432 & -4.15869 \\ \mathrm{O} & -3.00491 & 3.33231 & -1.55515 \\ \mathrm{~N} & -3.68480 & 1.74459 & -3.07951 \\ \mathrm{C} & -0.93848 & 2.08887 & 4.35085 \\ \mathrm{~N} & -0.33678 & -1.10774 & 5.20540 \\ \mathrm{C} & -3.74400 & 2.42300 & -1.89601 \\ \mathrm{~N} & -4.20023 & -0.62340 & -3.18282 \\ \mathrm{C} & -0.61181 & 0.20996 & 5.69990 \\ \mathrm{H} & -0.34634 & 0.28689 & 6.75770 \\ \mathrm{C} & -4.69690 & 0.72320 & -3.17993 \\ \mathrm{O} & -1.50101 & -2.85151 & 4.24798 \\ \mathrm{C} & -1.44139 & -1.71451 & 4.66442 \\ \mathrm{H} & -5.33226 & 0.90214 & -4.05119 \\ \mathrm{O} & -4.32988 & -2.49511 & -1.84388 \\ \mathrm{C} & -4.55186 & -1.32242 & -2.05589 \\ \mathrm{O} & -3.40964 & 3.55330 & 1.83810 \\ \mathrm{~N} & -2.17632 & 1.59165 & 4.64591 \\ \mathrm{~N} & -4.77991 & 1.91544 & -1.16610 \\ \mathrm{C} & -2.12403 & 0.38802 & 5.42294 \\ \mathrm{~N} & -2.47398 & -0.81204 & 4.70909 \\ \mathrm{H} & -3.11981 & 3.36168 & 4.23234 \\ \mathrm{C} & -5.44321 & 0.82631 & -1.82695 \\ \mathrm{~N} & -5.22760 & -0.46249 & -1.22794 \\ \mathrm{C} & -4.09526 & 2.57834 & 2.07944 \\ \mathrm{O} & -4.24730 & -2.37300 & 1.81193 \\ \mathrm{H} & -4.91874 & 3.60320 & -0.01390 \\ \mathrm{C} & -3.38506 & 2.31866 & 4.39777 \\ \mathrm{~N} & -4.11949 & 1.88388 & 3.25212 \\ \mathrm{C} & -5.32318 & 2.59159 & -0.02454 \\ \mathrm{H} & -2.73049 & 0.49240 & 6.32632 \\ \mathrm{~N} & -4.57896 & -1.22890 & 2.03740 \\ & -4.99359 & 1.99901 & 1.23265 \\ \mathrm{H} & -6.51153 & 1.03590 & -1.92388 \\ \mathrm{H} & -3.82170 & -1.17199 & 4.37302 \\ \mathrm{H} & -2.24621 & 4.19432 \\ \mathrm{H} & -0.41154 & 1.19151 \\ \mathrm{H} & 2.24196 & 5.28229\end{array}$




$\begin{array}{lrrr}\mathrm{C} & -5.87524 & -0.89899 & -0.02454 \\ \mathrm{H} & -5.81617 & -1.98613 & -0.00625 \\ \mathrm{H} & -6.41042 & 2.63212 & -0.13223 \\ \mathrm{C} & -4.99832 & 0.74843 & 3.21309 \\ \mathrm{C} & -5.63557 & 0.84362 & 1.80035 \\ \mathrm{H} & -4.47787 & -0.93849 & 5.21714 \\ \mathrm{H} & -6.92495 & -0.59173 & -0.05093 \\ \mathrm{H} & -5.72352 & 0.79657 & 4.02905 \\ \mathrm{H} & -6.72046 & 0.97454 & 1.81317\end{array}$

